\title{
A Survey on Brain Tumour Detection and Classification system based on Artificial Neural Network
}

\author{
Priya Kochar \\ M-Tech (Department of Computer Science) \\ Banasthali University, Jaipur, Rajasthan
}

\begin{abstract}
A Brain Tumour is one of the serious problems among various other existing life threatening diseases. Tumour detection is done initially by MRI, BIOPSY, SPINAL TAPE TEST ,ANNINOGARM and by some other similar kind of tests. All these tests are not only painful but are expensive too. Hence a brain tumour detection and classification system is required for early detection and categorization of tumour. In this paper we will study and analyze already proposed systems and will try to find the efficient and effective approaches. Tumour has a variant and complex structure and hence its classification is difficult .In the first phase Image pre-processing is performed initially on MR images of the patients to enhance features of brain cells and then a neural based classifier is implemented. BPNN, Radial basis and SMO based classifiers are examined. SMO when used with kmeans clustering provides a more accurate system. We have a learning phase where ANN is trained or learned by providing some images which are already classified as cancerous and non cancerous. After learning phase classification system is tested by giving some new inputs and comparing the results.
\end{abstract}

\section{Keywords}

Magnetic resonance Imaging (MRI), Back propagation network (BPNN), Sequential minimal optimization (SMO)

\section{INTRODUCTION}

Brain tumour is found to be one of the major problem in reducing life expectancy rate. Any human being whether a child or an adult can be affected by it. A tumour is a solid lesion which is formed due to abnormal growth of brain cells and it its symptoms varies on the location of tumour inside the brain. The average no of people who are suffering from various types of tumour is 87000 (2007) and this no. is growing rapidly. Brain is the central part of the body which is responsible for controlling and coordinating all other body organs, so if a tumour is located in a portion of brain then activities which are controlled by that part of nervous system are also affected.. Automated classification and detection of tumours in different medical images is motivated by the necessity of high accuracy when dealing with a human life. [3]. In this scenario medical image analysis and processing has a major influence in non-invasive treatment and clinical study as it provides new tools and techniques to doctors and researchers to diagnose the anomalies in the acquired images of the patients. Doctors can have a detailed and unambiguous internal structure of brain and can identify the abnormalities with more ease and understanding. Initially MRI of a patient is done which is a non -invasive medical test that helps physicians diagnose and treat medical conditions. It is a technique which depends on measurement of magnetic field vectors that are generated after appropriate excitation of strong magnetic field and radio frequency pulses in nuclei of hydrogen atoms present in water molecules of patients. [4] Magnetic resonance imaging (MRI), or nuclear magnetic resonance imaging (NMRI), is primarily a medical imaging technique used in radiology to visualize detailed internal structure and limited function of the body. To align the nuclear magnetization of (usually) hydrogen atoms in water in the body. Radio frequency (RF) fields are used to systematically alter the alignment of this magnetization. This causes the hydrogen nuclei to produce a rotating magnetic field detectable by scanner..Diagnoses of tumour was based on judgement of physicians after analyzing the images but there were more chances of false detection in these cases, hence digital image processing tools came into being which provides early and precise diagnose of tumour. Image segmentation is a process which best fits in this case. The main objective of image segmentation is to partition an image into mutually exclusive and exhaustive regions such that each region of interest is spatially contiguous and the pixels within the region are homogeneous with respect to predefined criteria [1]. The paper is organized in several sections. At first a brief introduction is given about tumour and existing approaches used by physicians. In second section digital image processing techniques are studied..Different ANN are discussed and implemented in section 3.Simulation and results are discussed in section 4 . The result of the paper and further improvements are studied in final section. We will study image classification first

\section{IMAGE CLASSIFICATION}

Image classification is done to separate the tumour affected part of image from rest of the image.

\subsection{Image Acquisition}

Images of the patient are acquired and stored at a common place. Some images are of normal patients that are not suffering from any disease and rest are abnormal brain images.. The images which are already categorized as cancerous and non-cancerous are used for reference and are used for checking the efficiency of proposed system. Images are scanned and checked by radiologists while going through MRI. The initial step after collection of MR image is to convert them in grey level and performing preprocessing.

\subsection{Image Pre-processing}

At first image pre-processing is done through Histogram equalization. Tumour has a dark appearance in the image so histogram equalization is used to make the edges visible. Irrelevant data is present in the medical images. 
Noise and inconsistencies can be removed and quality of image and enhancement of features are also fulfilled through image pre-processingtechniques.

\section{Histogram Equalization}

A method in which Histogram of an image is obtained and its contrast is adjusted is called as histogram equalization. Some images are having backgrounds and foregrounds with different intensities; In these cases this equalization technique can be applied. It is also known as spatial domain enhancement technique. [2].

\section{Thresholding}

It is the most basic method describing image segmentation. Here each pixel is associated with some value. A binary image is created by comparing the values of pixels with some threshold value and pixels are termed as object pixels if their value is greater than a threshold. An object pixel is assigned a value 1 and other a value 0 representing background. Black and white colours are assigned depending on pixel value and a binarized image is obtained. The process can be described by following equation:

$$
\mathrm{T}_{=} \frac{\sum_{i=0}^{M-1} \sum_{J=0}^{n-1} e_{i, j} * M_{I, J}}{\sum_{i=0}^{M-1} \sum_{J=0}^{N-1} M_{I, J}}
$$

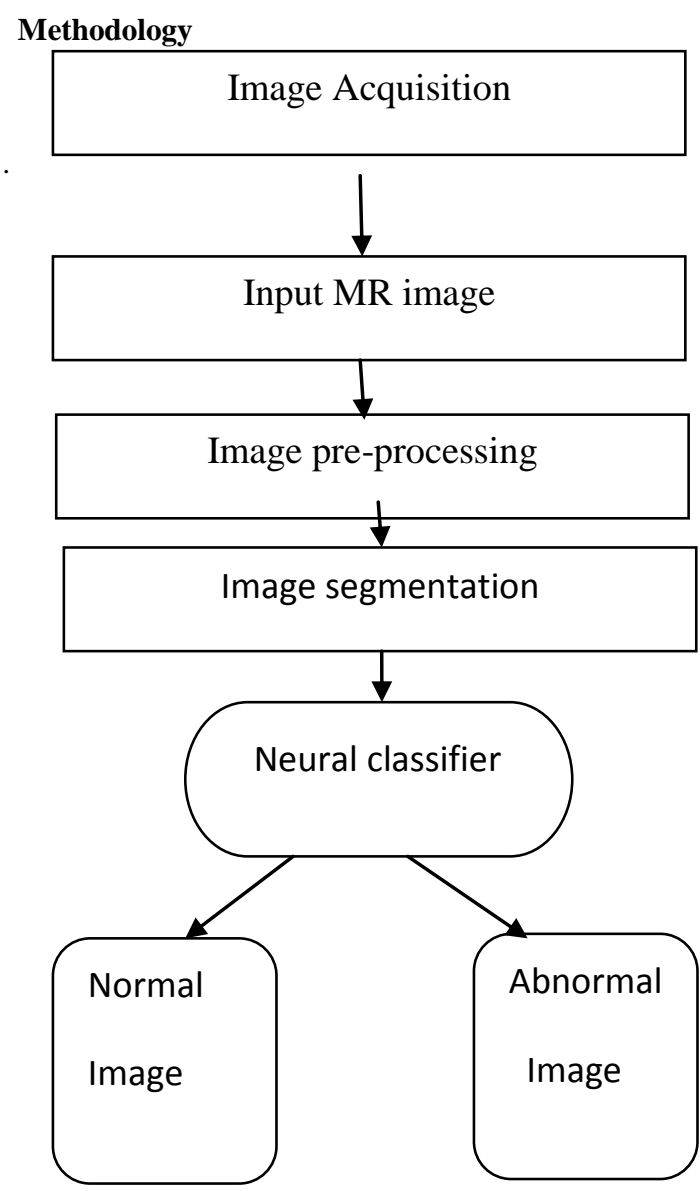

Each pixel's intensity value is compared with the threshold value and all the pixels having lesser value than the threshold are kept only and other pixels are removed. Every comparison is done starting from first row and first column. Given image contain salt and pepper noise which is removed in enhancement stage. The image shown in figure 2 contain the noise and in the subsequent image noise being removed and better appearance is achieved. This pre-processing step make the segmentation process more effective as the better contrast and improvement can be easily observed in the two images. The threshold value is represented by $\mathrm{T}$.

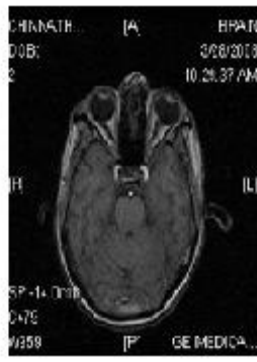

Before

Preprocessing

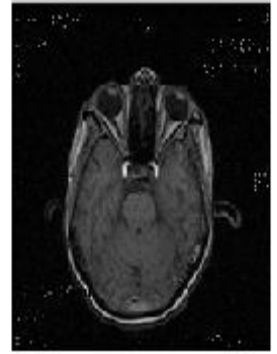

After
Figure: 2 Pre-processing [17 ]

\subsection{Image Segmentation}

Image segmentation is an important and challenging factor in digital image processing. Image segmentation is required to delineate the boundaries of the ROIs ensuring, in our case, that tumours are outlined and labelled consistently across subjects. Segmentation can be performed manually, automatically, or semi-automatically.[14] The manual method is time consuming and its accuracy highly depends on the domain knowledge of the operator. Specifically, various approaches have been proposed to deal with the task of segmenting brain tumours in MR images. In this process pixels are clustered in particular image regions. Location of tumours lesions and other abnormalities are processes which are found under region of interest identification. The proposed system works by first taking the images of normal patients and then acquiring important information from its anatomical structure and after that gathers information from unhealthy brain cell images. Every part of the reference image is compared with unhealthy brain images and abnormalities are detected.
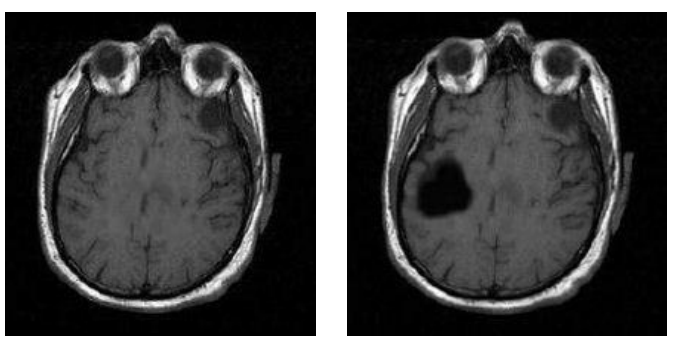

Figure:3 Normal brain

Figure 1: Basic architecture of system for classification of images 


\subsubsection{Image Enhancement}

Medical images are affected by noise and its degradations to a greater extent. Different types of noise is present in an image for e.g. salt and pepper noise ,impulse noise ,Gaussian noise etc. Denoising is done by using appropriate filters . Gaussian filter is used for removal of Gaussian noise .linear filters and average filters are also used.. On comparative analysis of work of researchers we have analysed that linear filters are not sufficient for enhancement when noise levels are too high. Sometimes a cascade of filters is required say Gaussian filter and average filter are used together for enhancement. Including the results of [1] Suppose a pixel of an image $I(x, y)$ is given and a random pixel $n(i, j)$ is added to it. Direct manipulation of pixels is done and

$g(x, y)=T[f(x, y)]$

in which $\mathrm{f}(\mathrm{x}, \mathrm{y})$ is the input image, $\mathrm{g}(\mathrm{x}, \mathrm{y})$ is the processed image, and $\mathrm{T}$ is an operator on $\mathrm{f}$, defined over some neighbourhood of $(\mathrm{x}, \mathrm{y})$. Gaussian filter In this paper, the proposed Gaussian smoothing filter, $g_{f}$, is a nonnegative, real-valued column matrix defined by[1]

$g_{f},(\mathrm{x}, \mathrm{y})=\frac{1}{c} \exp \left(\frac{-\left[x^{2}+y^{2}\right]}{2 \sigma^{2}}\right)$

Where $\mathrm{c}$ is $\mathrm{c}=\sqrt{2 \pi \sigma^{2}}$

This type of filters enhances the noise reduction

level compared with the linear filters, It was observed that these smoothing and noise filters did not completely satisfy the noise removal level from the original image. Thus, for these applications a set of cascaded filters are recommended. [1]
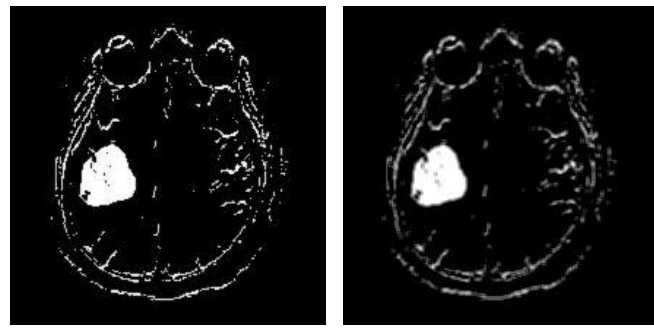

Figure:4 Gaussian filter Figure:5 Average filter [1]

\subsubsection{Edge detection}

The boundary between two regions with relatively distinct grey level properties is known as an edge. It is an important phase in image processing and computer vision [2]. Edges or boundaries of tumor are to be differentiated from normal brain cells. The purpose of edge detection in general is to significantly reduce the amount of data in an image, while preserving the structural properties to be used for further image -processing.[1]

\subsubsection{Clustering}

$\mathrm{K}$-means clustering algorithm is used to group the image in $\mathrm{k}$ clusters or $\mathrm{k}$ no of groups. In ANN learning is supervised and unsupervised and clustering is unsupervised learning. It is done by minimizing the Euclidean distance between data and corresponding cluster centroid[7] .Brain tissues are complex in structure having grey matter, white matter and cerebrospinal fluid ,hence localizing the region of interest has become quite difficult. As we have to form $\mathrm{k}$ clusters we at first need to select appropriate $\mathrm{k}$ centres .
Groups are formed by calculating each pixel's distance from available centre of clusters the pixel is assigned to the cluster whose centre is at minimum distance from it. after that centroid is again estimated. The algorithm suggested for application of k-means Algorithm

1 Choose no of clusters and assign them value like $\mathrm{k}$.

2 Arbitrarily choose $\mathrm{k}$ centres for the clusters.

3 Mean of each cluster is calculated

4 Compute each pixel distance with the chosen centres

5 Pixel is assigned to cluster which is at minimum distance.

6 Recomputed the mean of cluster after assigning the pixel .

Finally after implementing $\mathrm{k}$ means approach in the MR images tumour area is segmented in the form of separate cluster from rest of the images . A fuzzy c means algorithm is implemented in place of k-means to detect the appropriate shape of tumour and its classification.

\section{DESIGN OF ANN}

An artificial neural network (ANN), generally called neural network (NN), is a mathematical model or computational model that is inspired by the structure and/or functional aspects of biological neural networks. [8]. A neural network contains of an interconnected group of artificial neurons(processing element), working in unison to solve specific problems. ANNs, like people, learn by example. The neuron has two modes operations

The training/learning mode and the using/testing mode [16]. Neuron will fire only if

$\mathrm{X} 1 \mathrm{~W} 1+\mathrm{X} 2 \mathrm{~W} 2+\mathrm{X} 3 \mathrm{~W} 3+\ldots>\mathrm{T}$ The MCP neuron has the ability to adapt to a particular situation by changing its weights and/or threshold.

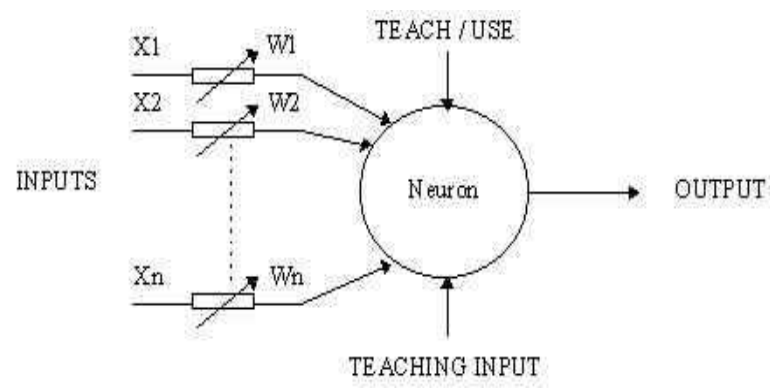

Figure6: Supervised learning [11]

\subsection{Back propagation Neural Network (BPNN)}

BP neural network is a multi-level error feedback network proposed by Rumelhart and Mc Clelladn in 1985. BP neural network architecture with one hidden layer operating on sigmoid transfer function has been employed for the classification of normal and abnormal tumour[9].In BPNN weights and biases are modified according to the desired output. if the actual output is not equal to desired 
output than the network is adjusted by modifying the weights.

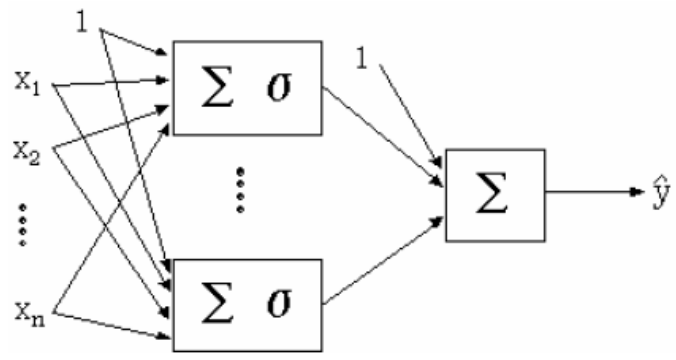

Figure7: BPNN [9]

Algorithm

1 Weights are initialized.

2 Inputs $x_{1}, x_{2} \ldots x_{n}$ moves layer by layer and a weigted sum with input vector is calculated.

3 If desired output is not equal to actual output than back propagation of error.

4 Weights and bias is updated accordingly.

The input and output of hidden layer neurons are calculate as [10]

$$
\begin{aligned}
& \operatorname{net}_{j}^{h}=\sum_{i=1}^{N+1} W_{J I} x_{i} \\
& y_{j}=\mathrm{f}\left(\text { net }_{j}^{h}\right)
\end{aligned}
$$

\subsection{Self Organizing Map}

The self organizing feature maps are neural networks which are based on competitive unsupervised learning. the output neurons compete among themselves to become activated [2] .The neuron in the output layer wining the completion is called winner take all neuron. the training phase is also called as vector quantization and neurons compete during training. Neural units in the competitive layer need to be approximately equal to the number of regions desired in the segmented image. It is not however, possible to determine a priori the correct number of regions in the segmented image. [1]. This has been the imitation of SOM .To overcome this limitation HSOM was implemented. HSOM combines the idea of regarding the image segmentation process as one of data abstraction where the segmented image is the final domain independent abstraction of input image $[1,16]$

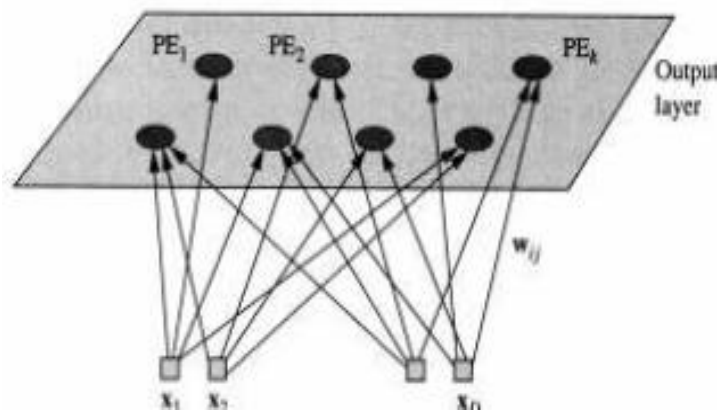

Figure $8 \mathrm{SOM}[2]$
According to Kohnen in this topographic map the coordinates of neurons in the lattice corresponds to intrinsic features of the input patterns. it means that the points close to each other in input space are mapped to same neighbourhood in output space

\subsection{Radial basis Neural Network}

The radial basis neural network was developed by Brrom head and law.

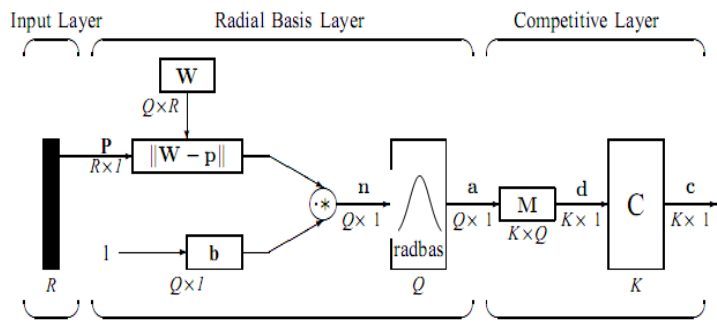

Figure 9 Radial basis layer in pnn model [3]

1)Input Layer: The input vector, denoted as $\mathbf{p}$, is presented as the black vertical bar i.

2) Radial Basis Layer: In Radial Basis Layer, the vector distances between input vector $p$ and the weight vector made of each row of weight matrix $\mathbf{W}$ are calculated. Here, the vector distance is defined as the product between two vectors

$$
\operatorname{radbas}(n)=e^{-n^{2}}
$$

the output of the network is a linear combination of radial basis functions of the inputs and neuron parameters. Radial basis functions have many uses including function approximations,.time series prediction. RBF networks have the advantage of not being locked into local minima as do the feed-forward networks such as the multilayer perceptron.

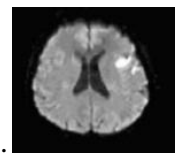

INPUT IMAGE

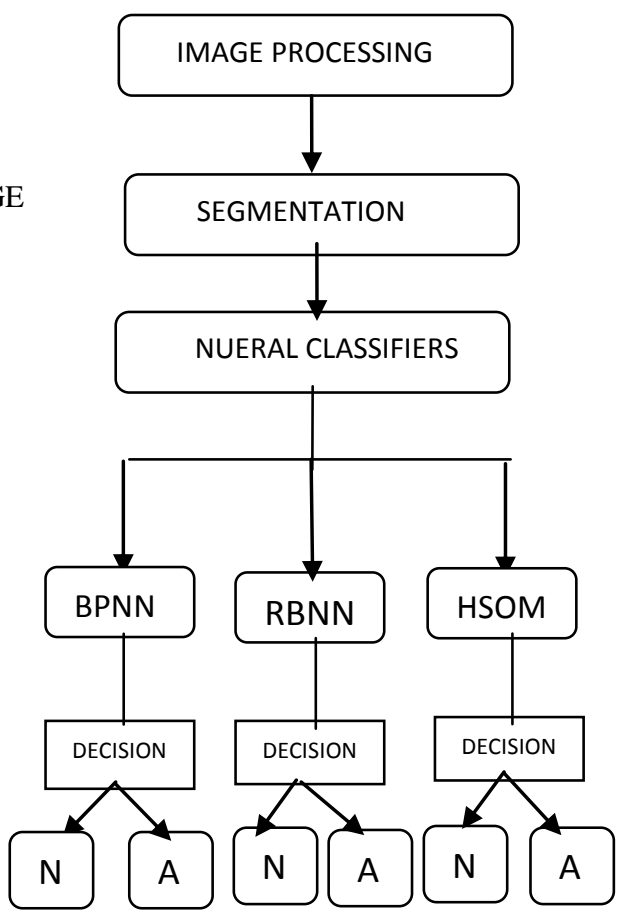

Figure 10 Proposed Classifier 


\section{SIMULATION AND RESULTS}

The image datasets are collected and implemented(mat lab). After applying image processing techniques they are implemented in neural classifiers as shown in figure. Several classifiers like feed forward neural network, BPNN ,Radial Basis NN,SMO etc are implemented for getting a better system .We will analyze BPNN first and the simulation results are taken for reference from[9]. Generalized windrow hoff learning rule is applied to this multilayered network with learning rate 0.5 and the value of activation function 0.5 activation function is used for mapping to a final output. Benign images are those with value less than 0.5 and higher values are considered for malignant images. The input data involved 42 patients (25 abnormal and 17 normal) The numbers of normal images for training set is 30 whereas for abnormal images are 12. Table 2 and Table 1 denote the performance evaluation of both classifiers. Table 2 denotes comparison in terms of sensitivity, specificity and accuracy. Sensitivity (true positive fraction) $=\mathrm{TP} / \mathrm{TP}+\mathrm{FN}$ Specificity (true negative fraction) $=\mathrm{TN} / \mathrm{TN}+\mathrm{FP}$ Accuracy $=\mathrm{TP}+\mathrm{TN} / \mathrm{TP}+\mathrm{TN}+\mathrm{FP}+\mathrm{FN}[9]$

Table 1 :Performance Of BPN [6]

\begin{tabular}{|l|l|l|l|}
\hline BPN & Normal & Abnormal & Total \\
\hline Prediction & $\mathrm{TP}=14$ & $\mathrm{FP}=7$ & 21 \\
\hline $\begin{array}{l}\text { False } \\
\text { negative }\end{array}$ & $\mathrm{FN}=3$ & $\mathrm{TN}=18$ & 21 \\
\hline Total & 17 & 27 & 42 \\
\hline
\end{tabular}

Table 2: Performance of RBNN

\begin{tabular}{|l|l|l|l|}
\hline BPN & Normal & Abnormal & Total \\
\hline Prediction & $\mathrm{TP}=15$ & $\mathrm{FP}=4$ & 19 \\
\hline $\begin{array}{l}\text { False } \\
\text { negative }\end{array}$ & $\mathrm{FN}=2$ & $\mathrm{TN}=21$ & 23 \\
\hline Total & 17 & 25 & 42 \\
\hline
\end{tabular}

Table 3: Final results

\begin{tabular}{|l|l|l|l|}
\hline Method & Sensitivity & Specificity & Accuracy \\
\hline BPN & $76.19 \%$ & $82.3 \%$ & $88.3 \%$ \\
\hline RBNN & $85 \%$ & $72 \%$ & $84 \%$ \\
\hline SMO & $92 \%$ & $90 \%$ & $89 \%$ \\
& & & \\
\hline
\end{tabular}

The available data is applied the SMO Based classification system..Each algorithm is trained and tested and the final analysis says that

\subsection{Implementation and Detection}

SMO with k-means clustering is most efficient among other classifiers. So result obtained by applying the classifiers results in classification of image as normal or abnormal..Tumour part in brain is highlighted in the image and clusters are shown.ref of these inputs is taken from[7]. Here the value of $\mathrm{k}$ is taken as 5.5 clusters are shown in the figure .

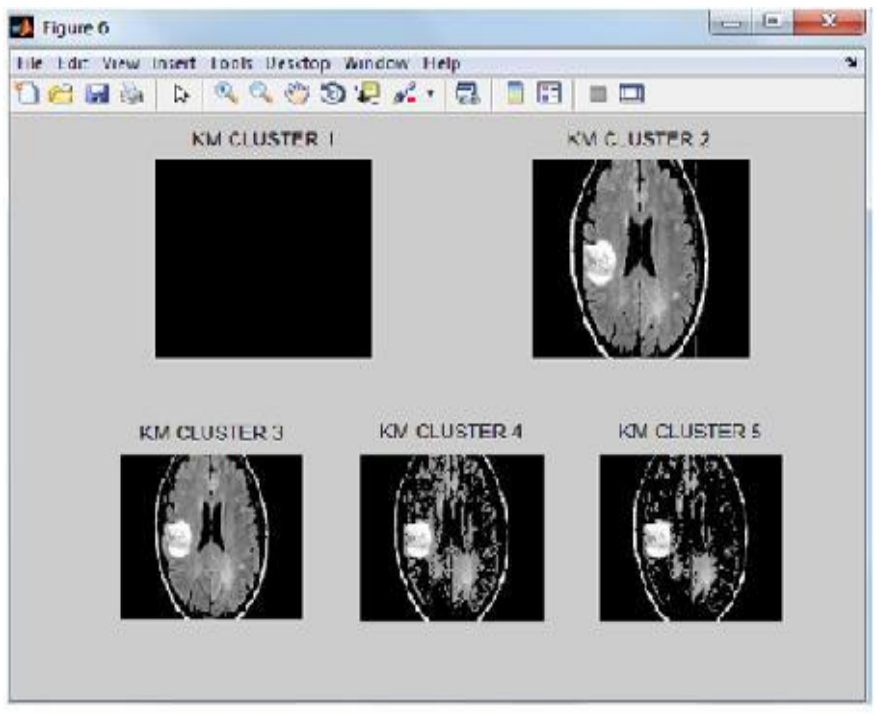

Figure 11 Output[ 7]

\section{CONCLUSION}

In this paper, we have proposed and studied three different approaches to classify the images as. The aim was to find the best approach so that an efficient system is developed. Further improvements can be achieved depending on the changes and other neural approaches. Neural when combined with fuzzy logic also gives an efficient classifier. The classifiers described in this paper categorizes the image as normal and abnormal and present the location of tumour via clustering. The manual procedure that pathologist choose for diagnosis is microscopic detection which is often time consuming and causes fatigue to them, hence this proposed system is quite beneficial . But there are several forms in which a tumour is categorized depending upon the size and location of tissues inside the 
the brain.. Some more samples of brain images should be collected possessing different grades of tumour in the training phase of the system .This SMO based classifier can be extended to a more efficient system by first training it with several images possessing benign, pre-maligant and malignant types of cancer and then in next phase it will be able to classify them in proper grades according to the provided samples. Hence the future work or further improvements in this system can be achieved by developing more detailed classifiers, efficient enough to categorize the brain the tumour depending on criticality of the tissues inside the brain .Only abnormality detection is not sufficient so achievement of this extension would be the next stepping stone in this field

\section{ACKNOWLEDGEMENT}

I express my deepest thanks to "Dr.Sumeet Gill" the mentor of the project for guiding and correcting various documents of mine with attention and care. He has taken the pain to go through the project and make necessary correction as and when needed. I also extended my heartfelt thanks to my family and well wishers.

\section{REFERENCES}

[1] Automated Brain Tumour Detection and Identification Using Image Processing and Probabilistic Neural Network Techniques. Dina Aboul Dahab1, Samy S. A. Ghoniemy2, Gamal M. Selim International Journal of Image Processing and Visual Communication. ISSN 2319-1724 : Volume (Online) 1 , Issue 2 , October 2012

[2] Brain tumour detection using unsupervised learning neural network. Ms Rungta college of engg and technology Bhilai..2013 international conference on Communication system and network technologies.

[3] Mohd Fauzi Othman ,'Probabilistic Neural Network For Brain Tumour Classification. University Technology Malaysia, 81310, Skudai, Malaysia.

[4] Carlos Arizmendi "s" Diagnosis of Brain Tumours from Magnetic Resonance Spectroscopy using Wavelets and Neural Networks". 32nd Annual International Conference of the IEEE EMBS Buenos Aires, Argentina, August 31 - September 4, 2010

[5] Azadeh Yazdan-Shahmorad MRSI Brain Tumour Characterization Using Wavelet and Wavelet Packets Feature Spaces and Artificial Neural Networks26th Annual International Conference of the IEEE EMBS San Francisco, CA, USA • September 1-5, 2004

[6] Dipali M. Joshi, Classification of Brain Cancer Using Artificial Neural Network 2010 2nd International Conference on Electronic Computer Technology (ICECT 2010)
[7] P Dhanlxmi," automatic segmentation of brain tumour using k-means clustering and its area calculation. CSE department ,ANNAMALI UNIVERSITY.

[8] Shweta JainBrain Cancer Classification Using GLCM Based Feature Extraction in Artificial Neural Network International Journal of Computer Science \& Engineering Technology (IJCSET) ISSN ISSN(PRINT):2278-8948,VOLUME2.ISSUE,2013

[9] S.N.Deepa \& B.Aruna Devi. Artificial Neural Networks design for Classification of Brain Tumour.

[10] Kailash D.Kharat1Brain Tumour Classification Using Neural Network Based MethodsGovt. Engineering College, Aurangabad, Maharashtra, India. International Journal of Computer Science and Informatics ISSN (PRINT): 2231 -5292, Vol-1, Iss-4, 2012

[11] Pankaj Sapra, Brain Tumour Detection Using Neural Network. International Journal of Science and Modern Engineering (IJISME) .ISSN: 2319-6386, Volume-1, Issue-9, August 2013

[12] AR Kvita An efficient approach for brain tumour detection based on modified region growing techniques. 2012 International Conference on Computing, Electronics and Electrical Technologies [ICCEET]

[13] An Artificial Neural Network Approach for Brain Tumour Detection Based on Characteristics of GLCM Texture Features in ann. International Journal of Computer Science \& Engineering Technology (IJCSE)

[14] Automated classification of Brain MRI using color converted K-means clustering segmentation and application of different kernel functions with multi class SVM. 1st Annual International Interdisciplinary Conference, AIIC 2013, 24-26 April, Azores, Portugal - Proceedings.

[15] S.Asif Hussain and M. Praveen Raju, "Neuron-Fuzzy System for Medical Image Processing"” Proceedings of the Inter. Conf. on Command Computational Intelligent., pp.382-385, India. Dec., 2010.

[16] Christos Stergion and Dimitrios Siganos, "Neural Networks", Pages 2-6. Shweta Jain / International Journal of Computer Science \& Engineering Technology (IJCSET)ISSN

[17] An Enhanced Implementation of Brain Tumour Detection Using Segmentation Based on Soft Computing 2010 International Conference on Signal Acquisition and Processing. 